\title{
A Complicated Course of Brain Tumor Resection in a Patient with a Left Ventricular Assist Device
}

\author{
Nimesh Patel ${ }^{1}$ Mohamed Fayed $^{1}{ }^{\circledR}$ Ahmed Ahmed ${ }^{1} \quad$ Akshatha G. Rao $^{1} \quad$ Derrick Williams $^{1}$ \\ Joseph A. Sanders ${ }^{1}$ \\ ${ }^{1}$ Department of Anesthesiology, Henry Ford Hospital, Detroit, \\ Michigan, United States \\ J Neuroanaesthesiol Crit Care 2022;9:115-118.

\begin{abstract}
Address for correspondence Nimesh Patel, MD, Department of Ford Hospital, 1350 West Bethune Street Apt 810, 48202, Detroit, Michigan, United States (e-mail: npatel25@hfhs.org).
\end{abstract} \\ Anesthesiology, Pain Management \& Perioperative Medicine, Henry
}

\begin{abstract}
Left ventricular assist devices (LVAD) are mechanical pumps that have become a standard treatment for end-stage heart failure. As patients with LVAD are living longer, the number of noncardiac surgeries performed in these patients is rising. However, these patients present a unique set of risk factors, some of which include acquired coagulopathies, anticoagulation status, and hemodynamic instability. Thus, performing noncardiac surgeries in patients with an LVAD requires a precise and complex surgical strategy with optimal communication among the surgical team. Therefore, knowledge of best perioperative approaches for patients with LVAD is urgently needed. Here, we present a detailed perioperative surgical approach in the case of a brain tumor

Keywords

- brain tumor resection

- coagulopathy

- left ventricular assist device

- hematoma resection for a 62-year-old patient with an LVAD whose course was complicated with a brain hematoma. Critical details include key aspects of monitoring patient hemodynamic stability and handling of anesthesia, patient positioning, and antiplatelet and anticoagulation drug therapy. This case highlights the importance for anesthesiologists to be well informed about perioperative LVAD management, as well as common complications that they may encounter.
\end{abstract}

\section{Introduction}

Approximately 550,000 cases of heart failure are diagnosed annually, leading to over US\$30 billion in healthcare expenditure in 2019. ${ }^{1}$ Left ventricular assist devices (LVAD), which are surgically implanted mechanical pumps, have become an integral aspect of treating end-stage heart failure. Studies have shown that intracranial hemorrhage is a common complication in patients with LVAD, with a presentation rate of 2.5 to $10 \%{ }^{2}$ We describe the unique management of a brain tumor resection in a patient who had long-term LVAD therapy.

\section{Case Report}

A 62-year-old woman, $(81.6 \mathrm{~kg}, 155 \mathrm{~cm})$ presented with 3 weeks of dizziness, ataxia, nausea, headaches, dysmetria, and upward gaze palsy. Brain imaging revealed a cerebellar tumor (-Fig. 1). Her medical history included breast cancer complicated with doxorubicin-induced cardiomyopathy for which HeartMate 3 was placed. Home medications included aspirin, furosemide, losartan, and warfarin. Her LVAD driveline site was clean and intact, and home LVAD parameters included a flow of $3.5 \mathrm{~L} / \mathrm{min}$, pump speed of $5250 \mathrm{rpm}$, pump power of 3.9 Watts, and pulsatility index (PI) of 8 . published online November 27, 2021
DOI https://doi.org/

10.1055/s-0041-1739347. ISSN 2348-0548. (c) 2021. Indian Society of Neuroanaesthesiology and Critical Care. All rights reserved.

This is an open access article published by Thieme under the terms of the Creative Commons Attribution-NonDerivative-NonCommercial-License, permitting copying and reproduction so long as the original work is given appropriate credit. Contents may not be used for commercial purposes, or adapted, remixed, transformed or built upon. (https://creativecommons.org/ licenses/by-nc-nd/4.0/)

Thieme Medical and Scientific Publishers Pvt. Ltd., A-12, 2nd Floor, Sector 2, Noida-201301 UP, India 


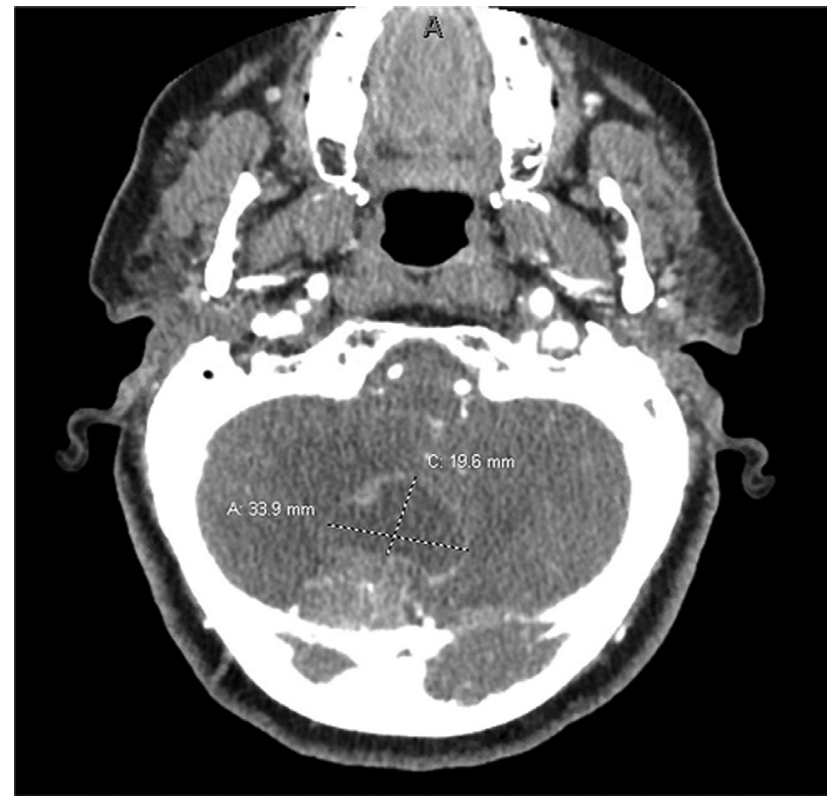

Fig. 1 Metastatic tumor in cerebellum causing compression of fourth ventricle and hydrocephalus. The patient had clinical symptoms of dysmetria and upward gaze palsy. Image reproduced with patient consent.

International normalized ratio (INR) at presentation was 2.79. Aspirin and warfarin were withheld for INR goal of less than 1.5. Heparin infusion as bridging anticoagulation therapy was started and paused 6 hours before surgery. Immediately before surgery, INR was 1.26 and other laboratory values were unremarkable. A preoperative discussion was held among the anesthesiologist, cardiologist, surgeon, and LVAD nurse.

Standard anesthesia monitors were applied. Multiple unsuccessful arterial cannulation attempts were made in the right radial, brachial, and axillary arteries before a left axillary arterial line was placed under ultrasound guidance. An 18G external jugular vein cannula was placed after failure of internal jugular vein visualization with ultrasound, and inability to pass guidewire beyond $16 \mathrm{~cm}$. Peripheral intravenous access included 20G right hand, 18G right foot, 18G right forearm, and Mediport catheter terminating in superior vena cava. Preoperative vital signs were 99\% oxygen saturation and mean arterial pressure (MAP) $95 \mathrm{mmHg}$. Intraoperative oxygen saturation was maintained at $99 \%$ and MAP was maintained between 80 and $85 \mathrm{mmHg}$. A mechanical circulatory support team, transesophageal echocardiogram (TEE), and vasopressor and inotropic drugs were readily available. Rapid sequence induction with GlideScope intubation was performed. Anesthesia was maintained with isoflurane 1 minimum alveolar concentration and remifentanil infusion at 0.08 to $0.1 \mathrm{mcg} / \mathrm{kg} / \mathrm{min}$. Ventilator settings were as follows: tidal volume $4 \mathrm{~mL} / \mathrm{kg}$, positive end-expiratory pressure (PEEP) 5, and fraction of inspired oxygen $60 \%$. Grounding pad was placed in the lower extremity to avoid mechanical interference. Bipolar cautery was used, and the surgeon was advised to use cautery in short bursts. During transportation, the LVAD was battery powered; in the oper- ating room, it was connected to the power base unit, which was powered by an electrical outlet.

The patient was positioned prone on gel rolls with her head secured to the Mayfield frame. Special attention was paid not to exert pressure onto the LVAD unit. Ephedrine $20 \mathrm{mg}$ was administered after positioning to correct a decreased blood pressure. Crystalloids (2.2 L) were administered without transfusions, $1.1 \mathrm{~L}$ of urine was produced, blood loss was $200 \mathrm{~mL}$, and LVAD parameters remained unchanged. After surgery, she was extubated and transferred to the intensive care unit in stable condition.

Subcutaneous heparin was started 48 hours after surgery as prophylaxis, and heparin infusion was restarted for warfarin bridging, targeting an INR of 2 to 3. On postoperative day 5 , the patient developed worsening headache. Brain imaging showed a cerebellar hematoma (-Fig. 2). Anticoagulation was stopped, protamine sulfate was administered, and the patient underwent uneventful emergency decompression and hematoma evacuation. On postoperative day 24 , she attained therapeutic INR and was discharged.

\section{Discussion}

LVAD placement in patients with heart failure is projected to grow as the number of older adults with heart disease increases in the United States. ${ }^{3}$ Aging is correlated with increased cancer risk $^{4}$; therefore, anesthesiologists may increasingly encounter LVAD patients with malignancy, and these patients have unique needs throughout the perioperative period.

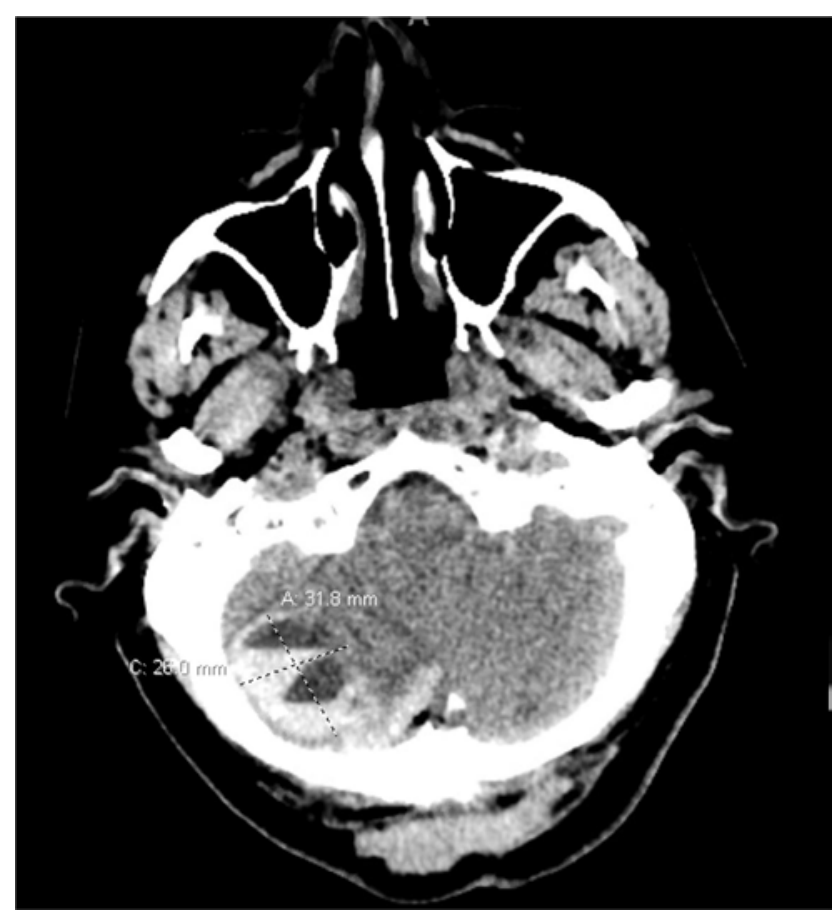

Fig. 2 On postoperative day 5, the patient developed a constant headache. Noncontrast head computed tomography showed a hematoma in the cerebellum with fourth ventricle compression and hydrocephalus. Image reproduced with patient consent. 
Regarding all LVAD devices, the anesthesiologist should be aware of the following specific LVAD parameters: (1) pump speed, (2) pump flow, (3) pump power, and (4) PI. Pump speed is fixed and is initially programmed by the implanting physician to optimize left ventricular unloading and ejection. A low fixed speed can cause increased risk for thrombosis, and a high fixed speed may cause hemolysis and left ventricular collapse through increased pump suction. Pump speeds for HeartMate 3 devices range from 3000 to $9000 \mathrm{rpm}$, but the clinically recommended range is 5000 to $6000 \mathrm{rpm}$. Power refers to the amount of energy required for the impeller to pump blood, which ranges from 4.5 to $6.5 \mathrm{~W}$. A low power may reflect low pump speed, an inflow cannula obstruction, or decreased preload. A high power can be due to increased preload, aortic insufficiency, or high pump speed. Pump flow refers to the amount of blood that is provided to the systemic circulation by the LVAD and ranges from 4 to $6 \mathrm{~L} / \mathrm{min}$. Pump flow is correlated with power and is affected by fixed speed, hematocrit, and the pressure gradient. Lastly, PI is a dimensionless index that averages the flow pulses (LVAD flow waveform) over 15 seconds with clinically normal values from 3.5 to 5.5. PI is increased with increased contractility and preload, and reduced with decreased preload and afterload or obstruction.

For our patient, general anesthesia was used without any issues. However, total intravenous anesthetic (TIVA) may have been the optimal choice, since the patient had a brain tumor with possible elevated intracranial pressure (ICP). Benefits of TIVA include decreased ICP and lowered cerebral metabolic rate oxygen consumption. The primary anesthesiologist felt that the volatile anesthetic would be more controllable for our patient. To optimize cerebral protection and decrease ICP, we used lidocaine during induction and hyperventilation, remifentanil infusion, mannitol $25 \mathrm{~g}$ intravenously, and head elevation during intubation, extubation, and transfer.

Patients with LVAD are coagulopathic. To prevent pump thrombosis, patients are given antiplatelet and anticoagulant therapy (target INR of 2-3). ${ }^{5}$ Risk of stroke in patients with LVAD is $~ 15 \%{ }^{6}$ High shear forces on blood flowing through the LVAD can lead to worsened coagulopathy. For nonemergent noncardiac surgery, anticoagulation is typically decreased to the lower limits of manufacturers' recommendations, except for neurosurgical or ophthalmological procedures that require full reversal. ${ }^{7}$ Patients presenting for emergent surgery can be given fresh frozen plasma, prothrombin concentrate, and vitamin K to reverse warfarin, or protamine sulfate for heparin. Intraoperative use of viscoelastic hemostatic assays can correct coagulopathy. However, clinicians must be aware that LVAD patients have altered baseline coagulation; thus, trends rather than absolute values should be followed. ${ }^{8}$ When postoperative bleeding risk is low, current guidelines recommend restarting heparin for partial thromboplastin time of 60 to $80 \mathrm{~s}$ until INR reaches 2 to 3 (level of evidence 1B). ${ }^{5}$

In neurological operations, MAP should be kept between 70 and $80 \mathrm{mmHg}$ to maintain cerebral perfusion in the setting of ICP. ${ }^{9}$ For LVAD patients, MAP should be less than $85 \mathrm{mmHg}$, as higher pressures can lead to pump stasis, flow reductions, and increased cerebrovascular accidents. Cere- bral oximetry can be used to monitor cerebral tissue oxygenation. In our patient, remifentanil infusion and volatile anesthetic were titrated for a MAP of $85 \mathrm{~mm} \mathrm{Hg}$ without any long-term postoperative neurological sequelae.

Patients with LVAD are preload dependent on right ventricle (RV) function, so preoperatively assessing RV function is important. Increased PEEP requirement during mechanical ventilation can increase airway pressures, further exacerbating RV dysfunction. Signs of RV failure are elevated central venous pressure, decreased urine output, increased capillary refill time, increased lactate, and end-organ dysfunction. Impaired RV contractility and leftward shift of the septum may be seen on TEE. LVAD patients may also experience device malfunction. Clinical signs are pump vibration, heart failure symptoms, decreased power and flow, inability to attain desired pump speed, and variable PI. TEE and mechanical circulatory support were available during our patient's treatment, but because the patient had stable hemodynamics and a functioning LVAD and to avoid surgery delay, preoperative TEE was not used.

Patient positioning can significantly affect hemodynamics in patients with LVAD. Prone positioning can cause decreased venous return leading to decreased preload and increased afterload as a result of arterial compression. Additionally, prone positioning might cause obstruction of the inflow cannula and create a suctioning event, compromising left ventricular end-diastolic filling and leading to systemic hypotension. ${ }^{10}$ Furthermore, prone positioning is associated with increased pulmonary artery pressure, which may reduce forward blood flow in patients with LVAD. Treating hypotension and assessing RV function with TEE are paramount should hemodynamic instability occur. In these situations, TEE could be placed after the patient is returned to supine position, or carefully while in the prone position.

\section{Conclusion}

Patients with an LVAD who require noncardiac surgeries pose a unique challenge to anesthesiologists. Our case highlights the importance of continually monitoring the LVAD patient's anticoagulation status and hemodynamic stability during surgery and being alert to possible postoperative complications. Clear communication between anesthesiologists, cardiologists, surgeons, LVAD nurses, and ancillary staff throughout the perioperative period is imperative.

Conflict of Interest

None declared.

Acknowledgments

We thank Karla D. Passalacqua, Ph.D. at Henry Ford Hospital for her critical review of the manuscript.

\section{References}

1 Benjamin EJ, Muntner P, Alonso AAmerican Heart Association Council on Epidemiology and Prevention Statistics Committee and Stroke Statistics Subcommittee. et al. Heart Disease and Stroke Statistics-2019 update: a report from the American Heart Association. Circulation 2019;139(10):e56-e528 
118 A Complicated Course of Brain Tumor Resection in a Patient with a LVAD Patel et al.

2 Factora FN, Bustamante S, Spiotta A, Avitsian R. Intracranial hemorrhage surgery on patients on mechanical circulatory support: a case series. J Neurosurg Anesthesiol 2011;23(01):30-34

3 John R, Holley CT, Eckman P, et al. A decade of experience with continuous-flow left ventricular assist devices. Semin Thorac Cardiovasc Surg 2016;28(02):363-375

4 White MC, Holman DM, Boehm JE, Peipins LA, Grossman M, Henley SJ. Age and cancer risk: a potentially modifiable relationship. Am J Prev Med 2014;46(Suppl 3):S7-S15

5 Feldman D, Pamboukian SV, Teuteberg JJ, et al. International Society for Heart and Lung Transplantation. The 2013 International Society for Heart and Lung Transplantation Guidelines for mechanical circulatory support: executive summary. J Heart Lung Transplant 2013;32(02):157-187

6 Kirklin JK, Naftel DC, Kormos RL, et al. Fifth INTERMACS annual report: risk factor analysis from more than 6,000 mechanical circulatory support patients. J Heart Lung Transplant 2013;32 (02):141-156

7 Stone M, Hinchey J, Sattler C, Evans A. Trends in the management of patients with left ventricular assist devices presenting for noncardiac surgery: a 10-year institutional experience. Semin Cardiothorac Vasc Anesth 2016;20(03):197-204

8 Kollmar JP, Colquhoun DA, Huffmyer JL. Anesthetic challenges for posterior spine surgery in a patient with left ventricular assist device: a case report. A Case Rep 2017;9(03):77-80

9 Vandse R, Papadimos TJ. Successful perioperative management of a patient with the left ventricular assist device for brain tumor resection: case report and review of the literature. Case Rep Anesthesiol 2015;2015:839854

10 Connors CW, Poltak JM, Christie AA. Noncardiac surgery in the prone position in patients with ventricular assist devices. J Cardiothorac Vasc Anesth 2012;26(01):e6-e7 